\title{
Arsenic relative bioavailability in contaminated soils: comparison of animal models, dosing schemes, and biological endpoints
}

\author{
H.B. $\mathrm{Li}^{1}{ }^{1}$ J. Li ${ }^{1}$, L.Q. $\mathrm{Ma}^{1}$ \& A.L. Juhasz ${ }^{2}$ \\ ${ }^{1}$ State Key Laboratory of Pollution Control and Resource Reuse, School of the Environment, \\ Nanjing University, Nanjing, P.R. China \\ ${ }^{2}$ Future Industries Institute, University of South Australia, Mawson Lakes, South Australia, Australia
}

\begin{abstract}
Different animals and biomarkers have been used to measure arsenic (As) relative bioavailability (RBA) in contaminated soils. However, there is a lack of comparison of As-RBA based on different animals (i.e., swine and mouse) and biomarkers [area under the blood As concentration curve (AUC) after a single gavaged dose vs. steady state As urinary excretion (SSUE) and As accumulation in liver and kidneys after multiple doses via diet]. In this study, As-RBA in 12 As-contaminated soils with known As-RBA via swine blood AUC model were measured using mouse blood AUC, SSUE, liver and kidneys analyses. Arsenic-RBA for the four mouse assays ranged $2.8-61 \%, 3.6-64 \%, 3.9-74 \%$, and $3.4-61 \%$, respectively. Compared to swine blood AUC assay (7.0-81\%), though well correlated $\left(\mathrm{R}^{2}=0.83\right)$, the mouse blood AUC assay generally yielded lower values. Similarly, strong correlations were observed between mouse blood AUC and mouse SSUE $(\mathrm{R} 2=0.86)$ and between urine, liver, and kidneys $\left(\mathrm{R}^{2}=0.75-0.89\right)$, suggesting As-RBA values were congruent among different animals and endpoints. Selection of animals and biomarkers would not significantly influence the role of in vivo assays to validate in vitro assays. Based on its simplicity, mouse liver or kidney assay following repeated doses of soil-amended diet is recommended for future As-RBA studies.
\end{abstract}

\section{INTRODUCTION}

Reliable assessment of human health risks from the ingestion of As-contaminated soil depends not only on total As concentration, but also its bioavailability. As such, various animal bioassays have been developed to quantify As relative bioavailability (RBA, relative to the absorption of sodium arsenate) in contaminated soils. As a measure of As-RBA, different biomarkers (As concentration in blood, kidneys, liver, or urine) have been used to determine As absorption following a single gavaged dose or multiple repeated doses of As-contaminated soil via diet. In addition to different animal models, varying feeding schemes have been employed to measure As-RBA in contaminated soil. While different animal models and feeding schemes have been used to assess As-RBA, comparative studies detailing the influences of these parameters on AsRBA measurement are lacking.

\section{METHODS/EXPERIMENTAL}

\subsection{Arsenic-contaminated soils}

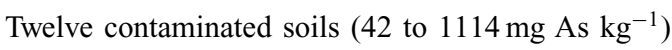
that had been measured for As-RBA using swine incorporating a single gavaged dose and area under blood As time curve (AUC) analysis were used in this study (Juhasz et al., 2009).

\subsection{Determination of As relative bioavailability using mouse bioassays}

Arsenic-RBA in the soils was determined using mouse bioassays using different dosing regimens and biological endpoints. Initially, As-RBA was assessed in fasted animals following administration of a single gavaged dose of sodium arsenate and soil suspension. Sodium arsenate was used as the reference dose. Blood samples of control and treated mice were collected at 4, $8,16,24$, and $48 \mathrm{~h}$ following gavage for As analysis to develop the blood As time curve. Arsenic-RBA was calculated by dividing the AUC following soil administration by the AUC following sodium arsenate administration following dose normalization.

In addition to AUC analysis, As-RBA in contaminated soils was also assessed using steady state As urinary excretion and As accumulation in liver and kidneys following multiple repeated As doses via diet. Prior to in vivo assays, soil and sodium arsenate were incorporated into the mouse basal diet. Each soil- or arsenate-amended diet ( $3 \mathrm{~g}$ each mouse per day) was provided to mice $(n=3)$ daily at 9 am for $10 \mathrm{~d}$. Following $8 \mathrm{~d}$ of exposure, mice were transferred to metabolic cages and urine collected each morning for the last two days of exposure and analyzed for As. At the end of the 10-d exposure period, the mice were sacrificed, and the liver and kidneys samples were collected for As analysis. When urine was used as the endpoint, As-RBA in soil was calculated as the ratio of the urinary As 


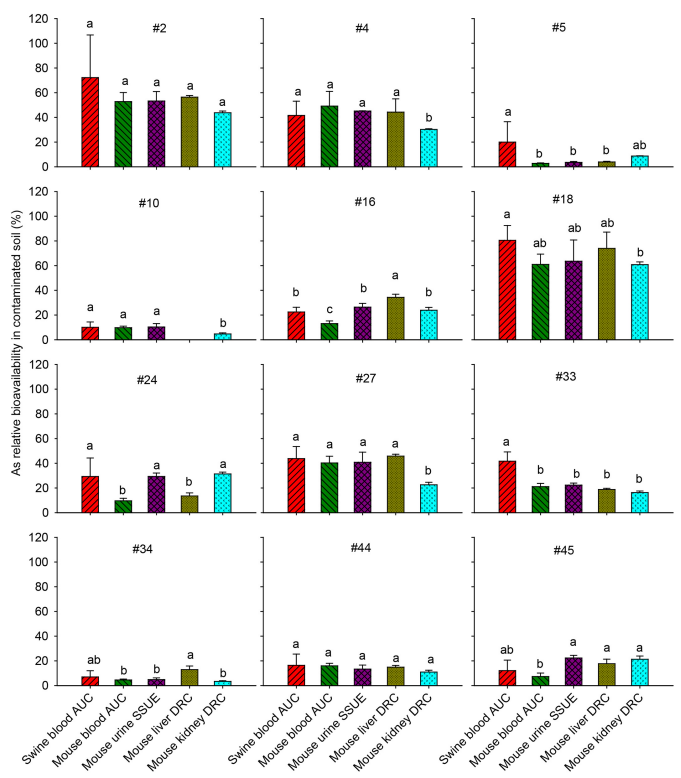

Figure 1. Arsenic relative bioavailability in 12 contaminated soils measured using swine blood AUC and mouse blood AUC models following a single gavage dose to fasted animals, mouse steady state urinary excretion (SSUE), and mouse liver and kidney dose response curve (DRC) models following multiple doses to soil-amended diets for $10 \mathrm{~d}$.

excretion fraction for soil exposure to that for sodium arsenate exposure. When liver or kidneys were used as the endpoint, Arsenic-RBA was calculated as the ratio of dose normalized As accumulation in liver/kidneys following 10-d exposure to soil-amended diet to that following 10-d arsenate-amended diet exposure.

\section{RESULTS AND DISCUSSION}

\subsection{Comparison of As-RBA by swine and mouse blood AUC bioassays}

Arsenic RBA in the 12-contaminated soils varied from 2.8 to $61 \%$ using the mouse AUC assay (Fig. 1). Compared to the swine AUC model, the mouse AUC assay generally yielded lower As-RBA for 11 out of the 12 soils. However, the differences between the two animal models were insignificant for most samples. A strong linear relationship $\left(\mathrm{R}^{2}=0.83\right)$ was observed for As-RBA based on swine and mouse models. The difference in As-RBA obtained by swine and mouse models was mainly caused by their different physiological structures, which affect As absorption.

\subsection{Comparison of As-RBA by different endpoints of mouse bioassays}

When urine was used as the biomarker of As exposure in mice, As-RBA in contaminated soils ranged from 3.6 to $64 \%$. When mouse liver and kidneys were used as exposure end points, As-RBA in contaminated soils ranged from 3.9 to $74 \%$ and 3.4 to $61 \%$, respectively, with generally lower values using kidneys. There was a significant correlation between As-RBA using kidneys and liver with $\mathrm{R}^{2}=0.75$. In addition, As-RBA determined using mouse liver and kidneys was correlated with that determined using urine as the endpoint $\left(\mathrm{R}^{2}=0.88-0.89\right)$.

\subsection{Comparison of As-RBA by different dosing approaches of mouse bioassays}

For AUC (single gavage dose) and SSUE (multiple doses via diet consumption) models, a strong linear relationship was observed $\left(\mathrm{R}^{2}=0.86\right)$. Similarly, strong linear correlations $\left(\mathrm{R}^{2}=0.88\right.$ and 0.69$)$ were found between As-RBA determined using the mouse AUC and mouse liver or kidney assays.

\section{CONCLUSIONS}

The results suggested that selection of different animals probably would not significantly influence the role of in vivo assays to validate in vitro assays. Though swine is the preferred animal model for As-RBA determination, mice can be used as a surrogate animal model due to its significant correlation with swine model in addition to its low cost and ease of handling.

In addition, different dosing approaches and endpoints did not significantly affect the in vivo-in vitro correlations. Compared to the single gavaged dose, which needs to establish the blood As time curve using large animal sizes, the repeated soil dose via diet offered the advantage of small numbers of animals required to determine As-RBA. For multiple dosing schemes, As in liver or kidneys is a simpler biological endpoint compared to urine. In short, based on this study, the mouse liver or kidney assay following repeated doses of soil-amended diet to mice is recommended for future As-RBA studies.

\section{ACKNOWLEDGEMENTS}

This work was supported by National Natural Science Foundation of China (21507057; 41673101).

\section{REFERENCE}

Juhasz, A.L., Weber, J., Smith, E., Naidu, R., Rees, M., Rofe, A., Kuchel, T. \& Sansom, L. 2009. Assessment of four commonly employed in vitro arsenic bioaccessibility assays for predicting in vivo arsenic relative bioavailability in contaminated soils. Environ. Sci. Technol. 43(24): 9887-9894. 